\title{
MEDIANS, LATTICES, AND TREES ${ }^{1}$
}

MARLOW SHOLANDER

In other papers $[3 ; 4]^{2}$ the author discussed the behavior of medians, segments, and betweenness in systems called median semilattices. A tree is a type of median semilattice. In this paper these systems are characterized in terms of a binary operation. It is further shown that these, along with more general systems, can be imbedded in distributive lattices.

The product notation in this paper is not to be confused with the betweenness notation of [4], nor is the ordered pair notation here to be confused with the segment notation there.

1. Semilattices. In this paper we consider sets $S$ which satisfy:

(K) $S$ is a semilattice.

This means that $S$ is closed under an idempotent, commutative, and associative multiplication $[1$, p. 18]. A necessary and sufficient condition [2] that $S$ be a semilattice is that it be closed under an idempotent multiplication which satisfies the cyclic-associative law $(a b) c$ $=(b c) a$. We say $a \leqq b$ if and only if $a b=a$. It follows that $S$ is a partly ordered set in which $x y$ serves as the greatest lower bound of $x$ and $y$. Conversely, a partly ordered set which contains with each pair of elements their greatest lower bound is a semilattice.

In a semilattice an upper bound of $a$ and $b$ is an element $t$ such that $a=a t$ and $b=b t$. A least upper bound, or join, of $a$ and $b$ is an upper bound $m$ of $a$ and $b$ with the property that $m t=m$ whenever $t$ is an upper bound of $a$ and $b$. It follows that if $a$ and $b$ have a join, it is unique and may be denoted by $a+b$. Similarly the join of a set of elements either does not exist or exists uniquely. The following theorem is easily proved.

(1.1) In a semilattice:

(i) $a+a$ exists and equals $a$.

(ii) $a+a b$ exists and equals $a$.

(iii) If $a+b$ exists, $b+a$ exists and is equal.

(iv) If $a+b,(a+b)+c$, and $(b+c)$ exist, then $a+(b+c)$ exists and equals $(a+b)+c$.

(v) If $a b+a c$ and $b+c$ exist, then $a b+a c \leqq a(b+c)$.

We list below additional postulates and consider relations between

Received by the editors June 8, 1953.

${ }^{1}$ This paper was written while the author was under contract to the Office of Ordnance Research.

${ }^{2}$ Numbers in brackets refer to the bibliography at the end of the paper. 
them. It proves convenient to state them in terms of joins. By a bounded pair of elements we mean two elements having the form $a b, a c$. Clearly $x$ and $y$ are a bounded pair if and only if they have a common upper bound $a$. A triple having the form $a b, b c, c a$ is called a mixed triple. If the existence of $b+c$ implies for all $a$ that $a b+a c$ exists and equals $a(b+c)$, we say that the join $b+c$ is preserved under multiplication. We use also the obvious extension of this terminology to joins of three or more elements.

(L) Joins of bounded pairs exist and are preserved under multiplication.

$\left(\mathrm{L}_{0}\right)$ Joins of mixed triples exist and are preserved under multiplication.

$\left(\mathrm{L}_{1}\right)$ The elements of a mixed triple are not distinct.

$\left(\mathrm{L}_{2}\right)$ Joins of pairs of elements exist and are preserved under multiplication.

$\left(\mathrm{L}_{3}\right)$ Elements $a, b$, and $a b$ are not distinct.

The past postulate is equivalent to the statement that $a b$ equals either $a$ or $b$. In the following theorems we assume, of course, that (K) holds.

(1.2) $\left(\mathrm{L}_{3}\right)$ is equivalent to $\left(\mathrm{L}_{1}, \mathrm{~L}_{2}\right)$.

Proof. Assume $\left(\mathrm{L}_{3}\right)$ holds. Given $a$ and $b$, we may assume $a b=a$. Then $a+b=a b+b=b$ and $x(a+b)=x b=x a b+x b=x a+x b$. Thus $\left(\mathrm{L}_{2}\right)$ holds. To prove $\left(\mathrm{L}_{1}\right)$, consider $a b, b c$, and $c a$ where, say, $a b=a$. If $a c=a, a c=a b$. If $a c=c, a c=a b c=b c$. Conversely, assume $\left(\mathrm{L}_{1}\right)$ and $\left(\mathrm{L}_{2}\right)$ hold and consider $a$ and $b$. From $\left(\mathrm{L}_{2}\right), a+b$ exists. From $\left(\mathrm{L}_{1}\right)$, the elements $a b, b(a+b)=b$, and $(a+b) a=a$ are not distinct.

(1.3) $\left(\mathrm{L}_{1}\right)$ and $\left(\mathrm{L}_{2}\right)$ each imply $\left(\mathrm{L}_{0}\right)$.

Proof. The second implication is trivial. Assume $\left(\mathrm{L}_{1}\right)$ holds. Consider $a b, b c$, and $c a$ where, say, $a b=b c$. Then both $a b$ and $b c$ equal $(a b)(b c)=a b c$. Hence the join of $a b, b c$, and $c a$ equals $c a$. Moreover $t$ times this join, $t c a$, is the join of $t a b, t b c$, and $t c a$.

(1.4) $\left(\mathrm{L}_{0}\right)$ implies (L).

Proof. Consider $a b$ and $a c$. Let $m$ be the join of the mixed triple $a(a b),(a b)(a c)$, and $(a c) a$. Then $m=a b+a c$.

An example showing that the converse of (1.4) need not hold is the set $\{a, b, c, a b+b c, b c+c a, c a+a b, a b, b c, c a, a b c\}$. The next two theorems restate known results and are easily proved.

(1.5) $\left(\mathrm{K}, \mathrm{L}_{2}\right)$ characterizes distributive lattices.

(1.6) $\left(\mathrm{K}, \mathrm{L}_{3}\right)$ characterizes chains.

2. Distributive semilattices. If a set $S$ satisfies Postulates K and L we call it a distributive semilattice. As noted above, $a+b$ exists in $S$ if and only if $a$ and $b$ have a common upper bound. 
The following theorem is a corollary of (1.1). Parts (iii), (iv), and (v) have alternate formulations. For example (iv) could read: If $a, b$, and $c$ have a common upper bound, $(a+b)+c=a+(b+c)$.
(2.1) In a distributive semilattice $S$
(i) $a+a=a$.
(ii) $a+a b=a$.
(iii) $a t+b t=b t+a t$.
(iv) $(a t+b t)+c t=a t+(b t+c t)$.
(v) $a(b t+c t)=a b t+a c t$.

This theorem could be summarized by saying that a bounded subset of a distributive semilattice is a distributive lattice. From (iv) it follows that an element is uniquely defined by expressions such as $a t+b t+c t+d t$. By (1.3) and (1.4) a semilattice for which $\left(\mathrm{L}_{0}\right)$ or $\left(\mathrm{L}_{1}\right)$ holds has the properties of (2.1). These properties are used freely in the following sections.

3. Median semilattices. Consider a set $S$ satisfying $\left(\mathrm{K}, \mathrm{L}_{0}\right)$. The join of $a b, b c$, and $c a$ is unique and may be denoted as $(a, b, c)$. Clearly $(a, b, c)$ is invariant under permutations of $a, b$, and $c$. From $\left(\mathrm{L}_{0}\right)$, it follows that $x(a, b, c)=(x a, x b, x c)$. We note $(a, a, b)=a+a b+b a=a$. This is Postulate $\mathrm{M}$ of [3]. We may also prove Postulate $\mathrm{N}$ holds. Let $(a, b, c)=r,(a, b, d)=s,(r, s, e)=x,(c, d, e)=t$, and $(t, a, b)=y$. We are to prove $x=y . y=t a+a b+b t$ where $t a=c d a+d e a+e c a$, and $b t=b c d+b d e+b e c$. That the expansion is valid follows from (2.1) and the fact that all terms used in each of these sums have $y$ as an upper bound. Similarly $x$ has an expansion which proves to be identical with that of $y$.

(3.1) $\left(\mathrm{K}, \mathrm{L}_{0}\right)$ characterizes median semilattices.

Proof. It remains to prove that $(\mathrm{M}, \mathrm{N})$ implies $\left(\mathrm{K}, \mathrm{L}_{0}\right)$. Choose an arbitrary element 0 in a set $S$ satisfying (M, N). Define $a b$ as $(a, 0, b)$. From $[3, \S 12]$, under this multiplication $S$ is a semilattice. From $(\mathrm{N})$ and $(8.7)$ of $[3], x(a, b, c)=(x a, x b, c)=(a x, b x, c x)$ and medians are preserved under multiplication. We see $m=(a, b, c)$ is an upper bound of $a b, b c$, and $c a$ since, for example, $a b(a, b, c)$ $=(a b a, a b b, c)=a b$. To show it is a least upper bound, consider an upper bound $t$ of $a b, b c$, and $c a . a m t=(a, a b, a c) t=(a, a b t, a c t)$ $=(a, a b, a c)=a m$. Similarly $b m t=b m$. Hence $m t=m(m t)=(a m t, b m t, c)$ $=(a m, b m, c)=m m=m$.

4. Trees. Consider a set $S$ satisfying Postulates $\mathrm{K}$ and $\mathrm{L}_{1}$. By (1.3) and (3.1), $S$ is a median semilattice. As in the proof of (1.3) we see that $a b=b c$ implies $(a, b, c)=c a$. Conversely, if $a b \neq b c$ we have, say, $a b=c a$ and $(a, b, c)=b c \neq c a$. Thus the following theorem holds. 
(4.1) $a b=b c$ if and only if $(a, b, c)=a c$.

(4.2) $(a, x, b),(b, x, c)$, and $(c, x, a)$ are not distinct.

Proof. Since each of the given medians has three possible values there are nine cases to consider. Suppose for example the medians equal respectively $a b, c x$, and $a x$. From (4.1) we have $a x=x b, b c=b x$, and $a c=c x$. Thus the medians have the respective values $a b, a c$, and $b c$ and they are not distinct. Other cases are treated similarly.

We have shown $\left(\mathrm{K}, \mathrm{L}_{1}\right)$ implies $\left(\mathrm{M}, \mathrm{N}, \mathrm{O}_{1}\right)$ of $[3]$. The converse implication, defining products as in (3.1), is trivial and the next theorem holds.

(4.3) $\left(\mathrm{K}, \mathrm{L}_{1}\right)$ characterizes trees.

5. An imbedding theorem. From the previous sections, we conclude a tree is a median semilattice and a median semilattice is a distributive semilattice. This section is devoted to a proof of the following theorem, which has as a corollary that median semilattices and trees can be imbedded in distributive lattices.

(5.1) A distributive semilattice can be imbedded in a distributive lattice.

Let $S$, the distributive semilattice, have elements $a, b, c, \cdots$. We use freely the properties listed in (2.1). Let $S_{1}$, the first extension of $S$, consist of the set of ordered pairs $(a, b)$ where $a$ and $b$ are in $S$. We define $(a, b)=(c, d)$ in $S_{1}$ if and only if in $S$ the following equations hold: $a=a c+a d, b=b c+b d, c=c a+c b, d=d a+d b$.

(5.2) The relation "=" in $S_{1}$ is an equivalence relation.

Proof. The properties of identity and symmetry are immediate. In proving transitivity assume $(a, b)=(c, d)$ and $(c, d)=(e, f)$. Then $a e+a f=a(e c+e d)+a(f c+f d)=a(e c+f c)+a(e d+f d)=a c+a d=a$. Three similar equations hold and hence $(a, b)=(e, f)$.

We define the product of $(a, b)$ and $(c, d)$ as $(a c+a d, b c+b d)$. This product has several forms since the following theorem is easily proved.

(5.3) $(x, y)=(y, x)$ and $(a c+a d, b c+b d)=(a c+b c, a d+b d)$.

(5.4) $S_{1}$ is a semilattice.

PROOF. That multiplication is idempotent and commutative is easily proved. The proof of associativity follows.

$$
\begin{aligned}
((a, b)(c, d))(e, f) & =(a c+a d, b c+b d)(e, f) \\
& =(a c e+a c f+a d e+a d f, b c e+b c f+b d e+b d f) \\
& =(a, b)(c e+c f, d e+d f)=(a, b)((c, d)(e, f)) .
\end{aligned}
$$

The following is readily proved.

(5.5) $(a, b)=(a, b)(r, s)$ if and only if $a r+a s=a$ and $b r+b s=b$. 
(5.6) $S_{1}$ satisfies Postulate L.

Proof. Let $(a, b)(r, s)=(a, b)$ and $(c, d)(r, s)=(c, d)$. We prove that $(a, b)$ and $(c, d)$ have a join $(x, y)$ where $x=a r+b r+c r+d r$ and $y=a s+b s+c s+d s$. First $(a, b)(x, y)=(a x+a y, b x+b y)=(a r+a s$, $b r+b s)=(a, b)$. Similarly $(c, d) \leqq(x, y)$. Next, if $(p, q)$ is an upper bound of $(a, b)$ and $(c, d)$, then $(x, y)(p, q)=(p x+q x, p y+q y)=(x, y)$. The previous equality holds since, for example, $p x+q x=r(a p+a q)$ $+r(b p+b q)+r(c p+c q)+r(d p+d q)=r a+r b+r c+r d=x$. Finally by routine computations we establish that for a given element $(e, f)$, by letting $z=a e+b e+c e+d e$ and $w=a f+b f+c f+d f$, we have $(a, b)(e, f)+(c, d)(e, f)=(z, w)=(e, f)(x, y)$.

From the formula for the join of $(a, b)$ and $(c, d)$ in the proof above it is seen that the join of $(v, v)$ and $(w, w)$ is $(v, w)$. Let $S_{1}^{*}$ be the subset of $S_{1}$ made up of pairs of the form $(x, x)$. The correspondence $x \rightarrow x^{\prime}=(x, x)$ is seen to be a one-one correspondence which preserves products. Thus $S$ is isomorphic to $S_{1}^{*}$ and in this sense (5.7) follows.

(5.7) $S_{1}$ is an extension of $S$. Any two elements in $S$ have a join in $S_{1}$. We now define $S_{n}$, the $n$th extension of $S$, as the first extension of $S_{n-1}$ for $n=2,3, \cdots$. We define $T$ as the union $S \cup S_{1} \cup S_{2} \cup \cdots$. Given a finite set of elements of $T$, there exists $k$ such that $S_{k}$ contains these elements. By induction on (5.4), $S_{k}$ is a semilattice and it follows that $T$ has the properties of a semilattice. Since any two elements in $S_{k}$ have a join in $S_{k+1}$, it follows that Postulate $\mathrm{L}_{2}$ holds and $T$ is a distributive lattice.

The extension $T$ of $S$ which has been described is minimal in the sense that a distributive lattice containing $S$ contains a sublattice isomorphic to $T$.

\section{BIBLIOGRAPHY}

1. Garrett Birkhoff, Lattice theory, Amer. Math. Soc. Colloquium Publications, vol. 25, rev. ed., 1948.

2. Lee Byrne, Bull. Amer. Math. Soc. vol. 52 (1946) pp. 269-272.

3. Marlow Sholander, Trees, lattices, order, and betweenness, Proc. Amer. Math. Soc. vol. 3 (1952) pp. 369-381.

4. ——, Medians and betweenness, Proc. Amer. Math. Soc. vol. 5 (1954) pp. 801-807.

WASHINGTON UNIVERSITY 